\title{
Demo Abstract: Demonstration of new sensor and actuator equipment for distributed grids
}

\author{
Marcus Meisel $^{1}{ }^{10}$ - Stefan Kollmann ${ }^{1}$ - Stefan Wilker ${ }^{1}$ - Alexander Wendt ${ }^{1}$. \\ Lampros Fotiadis $^{1}$. Friedrich Bauer ${ }^{1}$. Georg Kienesberger ${ }^{1}$
}

Published online: 30 August 2017

(C) The Author(s) 2017. This article is an open access publication

\begin{abstract}
Changes in the energy domain have created a high demand for new equipment and strategies to face its new challenges. To this end, stronger coordination between producers and consumers, as well as distributed control gain importance. This demonstration intends to show how developments from the project iniGrid can contribute towards this goal, by utilizing newly developed smart breakers to meet grid sided usage restrictions. The described demonstration system allows energy consumers more control over their usage and provides aggregators and energy suppliers as well as distribution system operators with additional means to improve grid stability and ways to counteract imminent catastrophic failures.
\end{abstract}

Keywords Smart grids - Distributed systems .

Local optimization $\cdot$ SCADA $\cdot$ CEMS

\section{Introduction}

Carbon emission reduction goals confront distribution system operators (DSOs) with new challenges, e.g. the introduction of high numbers of volatile, distributed energy

This paper is based on findings of the project Integration of Innovative Distributed Sensors and Actuators in Smart Grids, commissioned as flagship project by Österreischische Forschungsförderungsgesellschaft $m b H$ (FFG 4481266) as part of e!Mission.at, 4th call for proposals.

Marcus Meisel

marcus.meisel@tuwien.ac.at

1 Institute of Computer Technology, TU Wien, Gußhausstraße 27-29, 1040 Vienna, Austria producers. In face of such challenges, new approaches to handle energy generation and distribution emerge. Among these, researchers have identified a trend towards distributed control, e.g. Strasser et al. [1] does so regarding consumption and voltage optimization, while Faschang et al. [2] suggests a general development towards distributed control. Project iniGrid $^{1}$ leads this trend and aims to develop and test new sensor and actuator equipment, as well as providing application scenarios utilizing these. Meisel et al. [3] provides an overview of iniGrid.

\section{Setup}

The proposed demonstration will exemplify a producerdistributor-consumer setup by simulating interactions between a Supervisory Control and Data Acquisition (SCADA) system, a Customer Energy Management System (CEMS), local smart meters, and new smart breaker prototypes. Figure 1 shows a schematic overview of the components used in the demonstration where the SCADA system simulates the requests broadcast by energy providers (retailers or aggregators) and DSOs in one system, the CEMS mediating between their demands, and the users local requirements (eg. industrial processes, office hours). To this end, the CEMS is utilizing the available sensor and actuator equipment, i.e. the smart meter and smart breakers and, guided by a simple local intelligence, connect or disconnect local energy consumers to meet the remote system operators requests and demands. A photograph of the demonstration setup is shown in Fig. 2. The picture shows the first CEMS prototype on bottom right, implemented on a Raspberry Pi 3, the power socket next to it is switched by the central smart breaker in the hierarchical

\footnotetext{
${ }^{1}$ Project website: http://inigrid.at (accessed: 17.6.2017).
} 


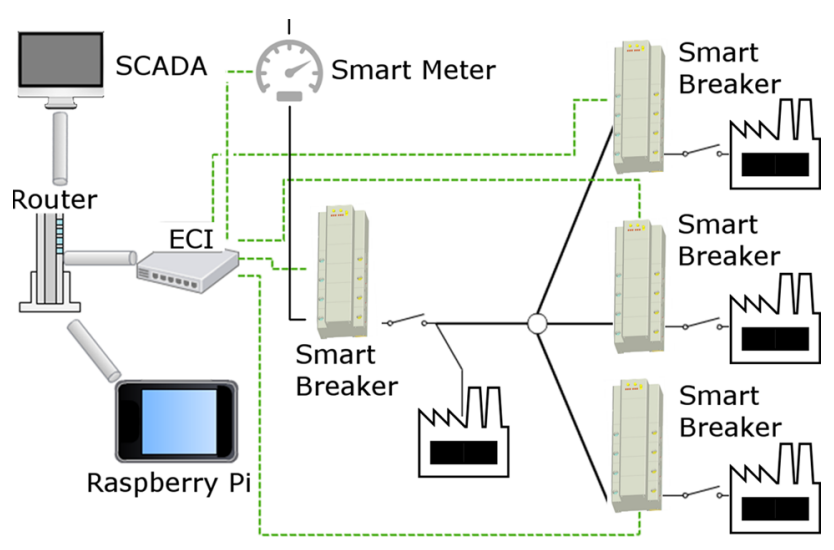

Fig. 1 Demonstration schematics

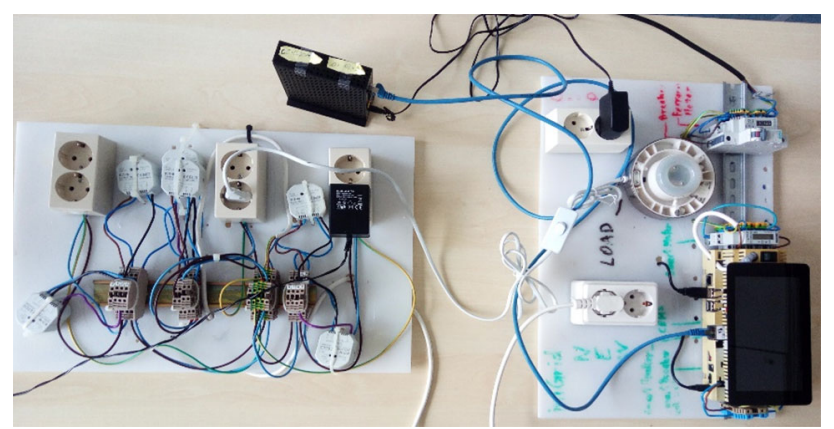

Fig. 2 CEMS setup

setup. The three remaining smart breakers are positioned on the second board.

\section{Scenarios}

Goal of this demonstration is to show how grid requirements could be implemented using smart sensors and actuators in a distributed way on the local level, benefitting all stakeholders. DSOs will have additional means to secure grid stability, ranging from presetting local limits reacting on small fluctuations, to new ways of avoiding total grid loss by increasing predictability and flexibility in emergency situations. We demonstrate the use of a traffic light based system that distinguishes between different levels of severity. Such a distinction allows the consumer to fine-tune CEMS reactions by differentiating devices switchable during minor grid fluctuations and more important ones only to be switched during times of imminent crises. Consumers can further customize the CEMS to respect device hierarchies, e.g. setups where disconnecting one machine only would render rest of the production line useless. This is demonstrated by broadcasting demands from the SCADA system, causing different enable/disable scenarios using the local smart breakers. Figure 3 shows one part of the SCADA user interface (UI): the

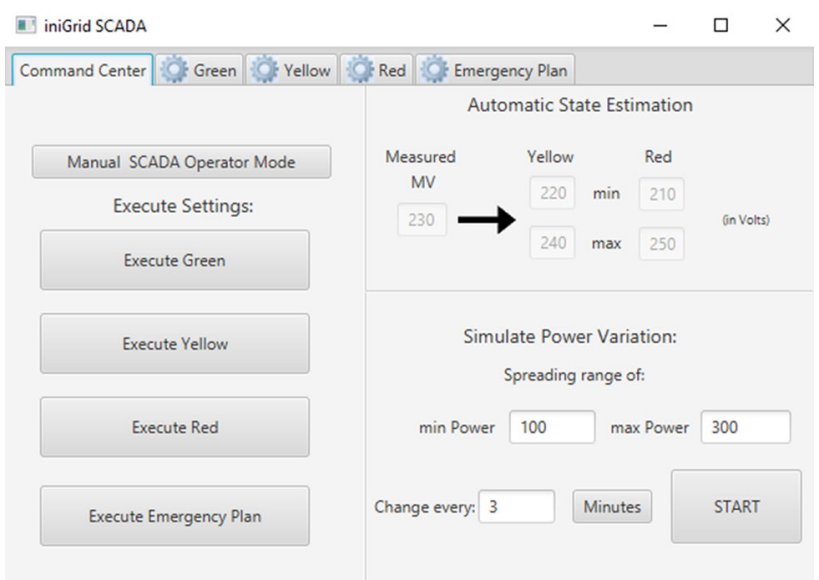

Fig. 3 Command center tab of SCADA system

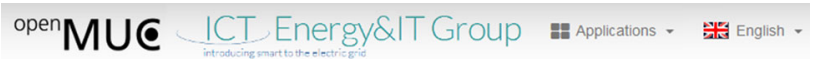

Power Meter Access Tool

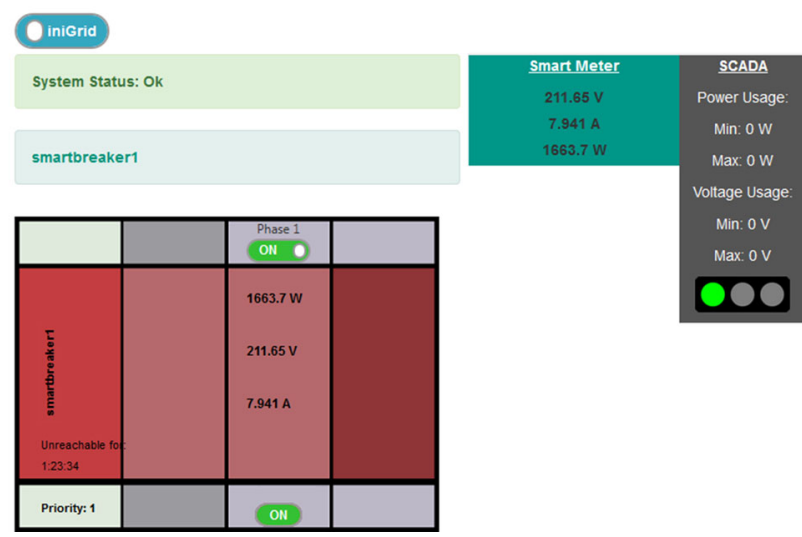

Fig. 4 CEMS user interface screenshot

command page that allows to switch between the different traffic light states. The demonstration uses security measures developed for these specific smart energy system use cases: a diode like approach implementing the DSO side broadcast to avoid CEMS to SCADA communication; use of IEC 62351, certificate based secure tunnels for communication as specified by the VHPready Alliance, fail safety implemented on the consumer side, proactively preparing for communication loss, and more. Figure 4 shows the CEMS UI for a smart breaker, displaying current smart meter measured consumption and restrictions of the SCADA system.

Acknowledgements Open access funding provided by TU Wien (TUW).

Open Access This article is distributed under the terms of the Creative Commons Attribution 4.0 International License (http://creativecomm ons.org/licenses/by/4.0/), which permits unrestricted use, distribution, and reproduction in any medium, provided you give appropriate credit 
to the original author(s) and the source, provide a link to the Creative Commons license, and indicate if changes were made.

\section{References}

1. Strasser T, Andrn F, Kathan J, Cecati C, Buccella C, Siano P, Leito P, Zhabelova G, Vyatkin V, Vrba P, Mařík V (2015) A review of architectures and concepts for intelligence in future electric energy systems. IEEE Trans Ind Electron 62(4):2424-2438
2. Faschang M, Xypolytou E, Meisel M, Wendt A, Kaufmann T, Litzlbauer M, Marchgraber J, Bibl M, Prostejovsky A, Gawron-Deutsch T, Kienesberger G (2015) Transition roadmap-from centralized to massively decentralized grid control systems, technical report for FFG, Eigenverlag, p 75. ISBN: 978-3-200-04337-4

3. Meisel M, Wilker S, Wess M, Wendt A, Sauter T, Kienesberger G (2016) ICT emulation platform setup demonstration of smart grid component prototype examples. In: 21st IEEE international conference on emerging technologies and factory automation ETFA 2016, 6-9 Sept., Berlin, Germany 